\title{
Leyes básicas del comportamiento físico de los suelos con y sin cohesión
}

\author{
L. Zeevaert-Wiechers \\ División de Estudios de Posgrado, Facultad de Ingeniería, UNAM
}

(recibido: marzo de 2001; aceptado: junio de 2001)

\begin{abstract}
Resumen
Para el conocimiento racional del comportamiento de los suelos, es necesaria la investigación reológica de las características índice y mineralógicas básicas de los suelos que se representan en la naturaleza.

Así también, para conocer la propiedad cuantitativa física se hace necesario el conocimiento de las leyes físicas que lo gobiernan y la aplicación de éstas a su comportamiento.

Además, tiene gran importancia la correcta investigación de los parámetros que los rigen y que deben adecuarse al comportamiento de los suelos en campo, por medio del conocimiento estratigráfico, la observación y el análisis en el laboratorio.
\end{abstract}

Descriptores: comportamiento de los suelos, reología, propiedades, leyes físicas, estratigrafía.

\begin{abstract}
To be able to obtain a rational understanding of the soil behavior, it is necessary to perform a rheologically-oriented investigation of the basic index and mineralogical characteristics of the soils found in nature. On the other hand, to determine the quantitative physical properties it is also important to have a knowledge of the governing physical laws and the application of these laws to the soil behavior. Finally, it is of utmost significance to have a proper assessment of the parameters that govern the soils which in turn have to be adjusted to their behavior in the field through the understanding of the stratigraphy, their observation and their analysis in the laboratory.
\end{abstract}

Keywords: soil behavior, rheology, properties, physical laws, stratigraphy

\section{Introducción}

En la naturaleza se encuentran suelos formados en diferentes ambientes, que generaron determinado estructuramiento físico particular, con diferentes compresibilidades y resistencias, las cuales dependen del arreglo estructural durante su formación ambiental.

Se encuentran desde estructuras simples de suelos granulares poco cohesivos con sensibilidades de 1.5 o menor, hasta suelos lacustres floculentos o doblemente floculentos de alta compresibilidad con sensibilidades de 15 o más. Para estimar en forma tentativa el grado de compresibilidad, se puede analizar la relación que existe entre la resistencia no confinada del suelo inalterado con la resistencia del mismo suelo que ha sido amasado o remoldeado sin perder el grado de saturación natural, esto es $\mathrm{S}_{\mathrm{r}}=\mathrm{q}_{\mathrm{u}} / \mathrm{q}_{\mathrm{r}}$, (Zeevaert, 1998).
Sin embargo, todos los suelos se relacionan con las características que corresponden a una formación ambiental definida y se distinguen por tener características típicas básicas, a saber:

1. Suelos residuales

2. Suelos eólicos

3. Suelos lacustres

4. Suelos marinos

5. Suelos aluviales

6. Suelos de piemonte

Todos estos suelos, ya sean clásticos o piroclásticos, se pueden encontrar en la naturaleza semi-saturados, o bien, saturados, excepto los eólicos, que siempre se encuentran con un porcentaje bajo de saturación. Al saturarse, estos suelos sufren colapso en su estructura ( $V$ an, 1983). 
Los suelos residuales y lacustres pueden sufrir hundimiento o contracción de diferente intensidad, dependiendo del aumento o disminución del grado de saturación y del mineral de arcilla que contengan (Zeevaert, 1988).

Los suelos marinos tienen características diferentes a otros suelos terrestres, tienen granulometrías específicas provocadas por la sedimentación marina se encuentran con y sin cohesión.

Los suelos aluviales y de piemonte son de granulometría gruesa comparados con los suelos descritos anteriormente; por tanto, no representan en general los fenómenos que sufren en su constitución estructural los suelos que contienen mayor proporción de minerales de arcilla. Zeevaert (1997) y (1998)

Por consiguiente, el conocimiento de las características índice y propiedades mecánicas y mineralógicas de los granos que forman la estructura del suelo, son importantes para su clasificación y ubicación dentro del contexto de la mecánica de suelos para poder pronosticar en forma tentativa su probable comportamiento típico. (Figura 1) Zeevaert (1998) y (1985).

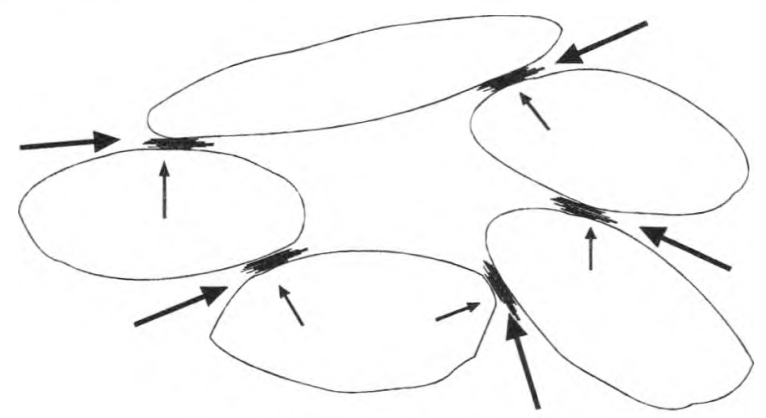

ESFUERZO TANGENCIAL

\section{Figura 1. Esfuerzos entre granos en la estructura del suelo}

Para poder conocer el comportamiento cuantitativo físico de los suelos, el ingeniero investigador tiene que familiarizarse con las leyes físicas básicas del comportamiento reológico de los materiales, y aplicarlas a los suelos, permitiendo establecer con las leyes dicho comportamiento físico cuantitativo. Zeevaert (1997) y (1998), (Terzaghi , 1941).

Se hace hincapié en la importancia que tiene el conocimiento físico de los granos del suelo en cuanto a su dureza, dimensiones y formas, así como de los minerales de arcilla que se encuentran rodeados de agua altamente viscosa, incluso cristalizada. Esta agua sólida o agua altamente viscosa, liga fuertemente entre sí a los granos con espesores atómicos. Lo anterior es muy importante en el comportamiento de los suelos arcillosos, ya que esta situación se puede investigar por métodos que existen en la reología y en la físico-química de los suelos; su clasificación es muy importante en los análisis específicos de estos suelos que se encuentran en la naturaleza, como el caso de los depósitos de arcilla limosa, doblemente floculenta típica de la ciudad de México DF. Zeevaert (1997) y (1998).

Las leyes físicas básicas que se aplican al estudio de los sólidos, son también aplicables a los suelos en general.

\section{Leyes reológicas}

Ley de elasticidad (Hooke, 1670) $\gamma=\alpha \tau$ o bien $\varepsilon=\beta \sigma$

Aquí:
$\gamma \quad$ Distorsión angular
$\tau \quad$ Esfuerzo cortante
$\varepsilon$ Deformación unitaria, lineal o volumétrica
$\sigma \quad$ Esfuerzo unitario, lineal o volumétrico
$\alpha, \beta$ Son parámetros constantes que dependen de la temperatura, del esfuerzo octaédrico a que esté sometido el suelo en campo, así como de su densidad durante la acción.

La elasticidad pura se reconoce porque el material se deforma instantaneamente con la aplicación del esfuerzo y al retirar dicho esfuerzo el material se repone totalmente de la deformación; este fenómeno no es función del tiempo:

"La elasticidad proporciona la estabilidad".

Ley de plasticidad y resistencia (Coulomb, 1783) $\rho=\mu \sigma$

Aquí:

$\rho \quad$ Esfuerzo cortante unitario último para ocasionar la falla

$\sigma \quad$ Esfuerzo unitario normal a la superficie de falla

$\mu$ Parámetro de la fricción, es función de la temperatura y esfuerzo octaédrico a que se encuentre sujeto el suelo en campo, de la oquedad y forma de los granos.

La acción de la plasticidad es independiente del tiempo, se genera por el resbalamiento instantáneo relativo entre granos de la estructura del suelo y el resbalamiento total a lo largo de una superficie, provoca la falla según la ley de Coulomb, así cuando se retira la carga, el suelo no recupera su condición geométrica inicial.

Cuando hay estabilidad en el proceso plástico, ésta es proporcionada por los elementos elásticos con relación a 
los elementos plásticos que contenga el suelo. El fenómeno no depende del tiempo, algunos suelos no saturados poseen la elasto-plasticidad adicionada de flujo plástico (Zeevaert, 1994). (Figura 2 $2^{\mathrm{a}}$ y 2 b).

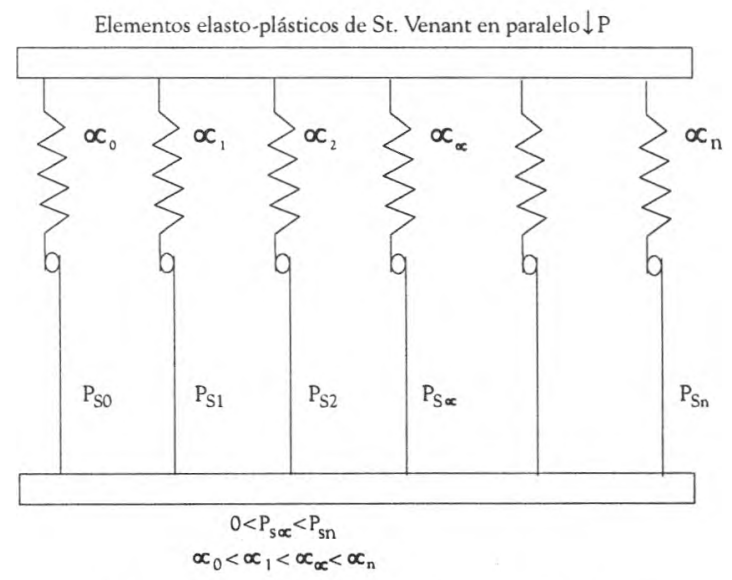

Figura 2a. Modelo elasto-plástico

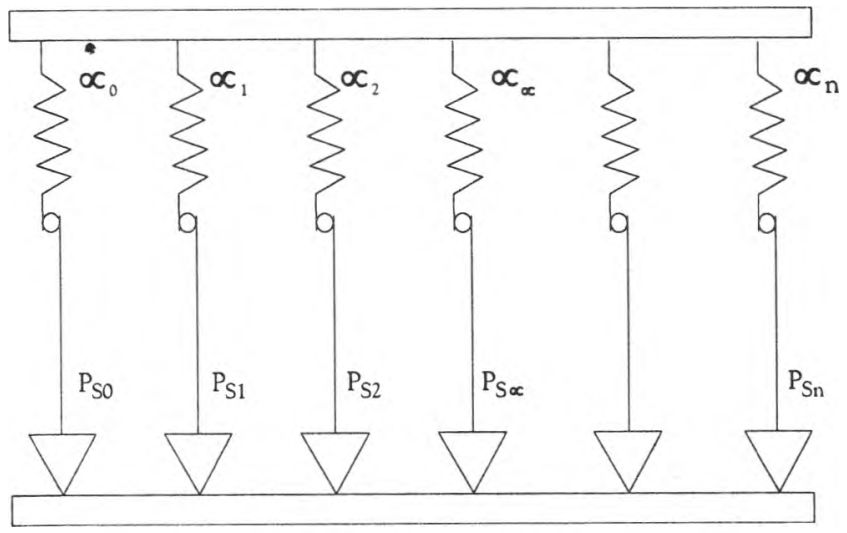

Figura 2b. Modelo elasto-plástico con fluidez (viscosidad)

Ley de fluidez (Newton, 1685) $\dot{\gamma}=\phi \tau$, o bien $\dot{\varepsilon}=\phi \sigma$

Aquí:

$\gamma \quad$ Distorsión angular respecto al tiempo

$\tau$ Esfuerzo cortante que permanece constante durante la acción

$\dot{\varepsilon} \quad$ Deformación unitaria respecto al tiempo

$\sigma$ Esfuerzo efectivo que permanece constante durante la acción

$\phi$ Parámetro que representa la fluidez, (inverso de la viscosidad) es función de la temperatura, del tiempo y esfuerzo octaédrico a que se encuentre sujeto el suelo en el campo y su densidad.
La fluidez o flujo plástico se reconoce porque a través del tiempo la deformación del suelo continúa indefinidamente, por consiguiente, la fluidez es una función del tiempo gobernada por el parámetro $\phi_{1}$.

La velocidad de la deformación unitaria es proporcional al esfuerzo y a la temperatura, los cuales permanecen constantes durante la acción. El parámetro $\phi_{1}$ se presenta con diferentes valores a temperatura constante, a saber:

C1. $\phi_{0}$ Constante. Que representa al cuerpo newtoniano lineal

C2. $\phi$ - Deformación decreciente con el tiempo $\left(\phi_{0}+\alpha /(1+T)\right)$

C3. $\phi+$ Deformación creciente con el tiempo $\left(\phi_{1}+\beta T\right)$

La figura 3 muestra configuraciones típicas del fenómeno de la fluidez en los suelos viscosos de acuerdo con los parámetros físicos C1, C2 y C3, que se indicaron anteriormente. El flujo plástico se observa en todos los suelos con diferente intensidad, los suelos que presentan mayor intensidad en la viscosidad son los cohesivos, los cuales contienen minerales de arcilla de alta actividad como son los del grupo de las montmorilonitas.

Por medio de la combinación de las leyes básicas, se establecen unidades reológicas para diferente comportamiento de los suelos. En la tabla 1 se muestran las unidades reológicas básicas que se utilizan para representar el comportamiento físico cuantitativo para cada suelo en cuestión. Además, es necesario determinar en el laboratorio, en muestras inalteradas representativas, los parámetros que los rigen y que correspondan a las condiciones ambientales en el campo.

El estudioso puede analizar unidades reológicas; por ejemplo, la de St. Venant en paralelo, que muestra el comportamiento de los suelos no saturados. Si adiciona las unidades Newtonianas se puede representar el comportamiento a través del tiempo de los suelos no saturados. (Figura $2 \stackrel{a}{a}$ y $2 b$ ).

La siguiente fórmula nos muestra el comportamiento de este tipo de suelos no saturados, confinados en la naturaleza. Figura 3 (Zeevaert, 1994).

$$
\left.\varepsilon=\mathrm{m}_{\mathrm{ep}}(\sigma)+\alpha(\sigma) \log (\mathrm{b}+\mathrm{t}) / \mathrm{b}\right)
$$

Aquí:

$\varepsilon \quad$ Deformación unitaria

$\sigma \quad$ Esfuerzo unitario

t Tiempo

$\mathrm{m}_{\mathrm{ep}}$ Módulo de compresibilidad volumétrica elastoplástico 
DOI: http://dx.doi.org/10.22201/fi.25940732e.2001.02n3.012

Tabla 1. Unidades Reológicas para los suelos

1. Hook (1670) Elasticidad

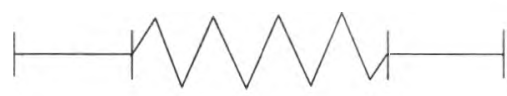

2. Coulomb (1783) Resistencia y plasticidad

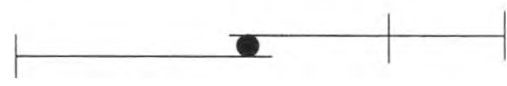

3. Newton $(1685) \quad$ Fluide $z=c /$ viscosidad

a) Fluidez lineal

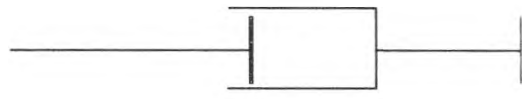

b) Fluidez decreciente con el tiempo

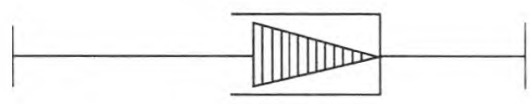

c) Fluidez creciente con el tiempo

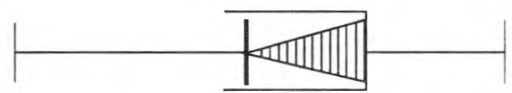

4. St. Venant $=$ Hook en serie con Coulomb

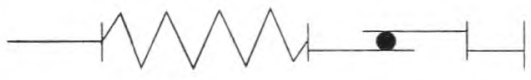

5. Maxwell $=$ Hook en serie con Newton

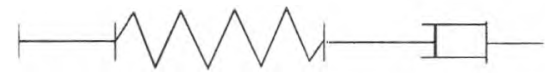

6. St. Venant en serie con Maxwell

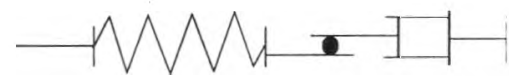

7. Kelvin $=$ St. Venant en paralelo con Newton $=$ Terzaghi

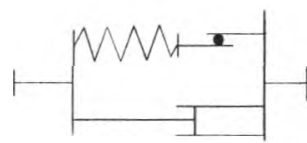

8. Unidad "Z" = Newton lineal con Newton no lineal
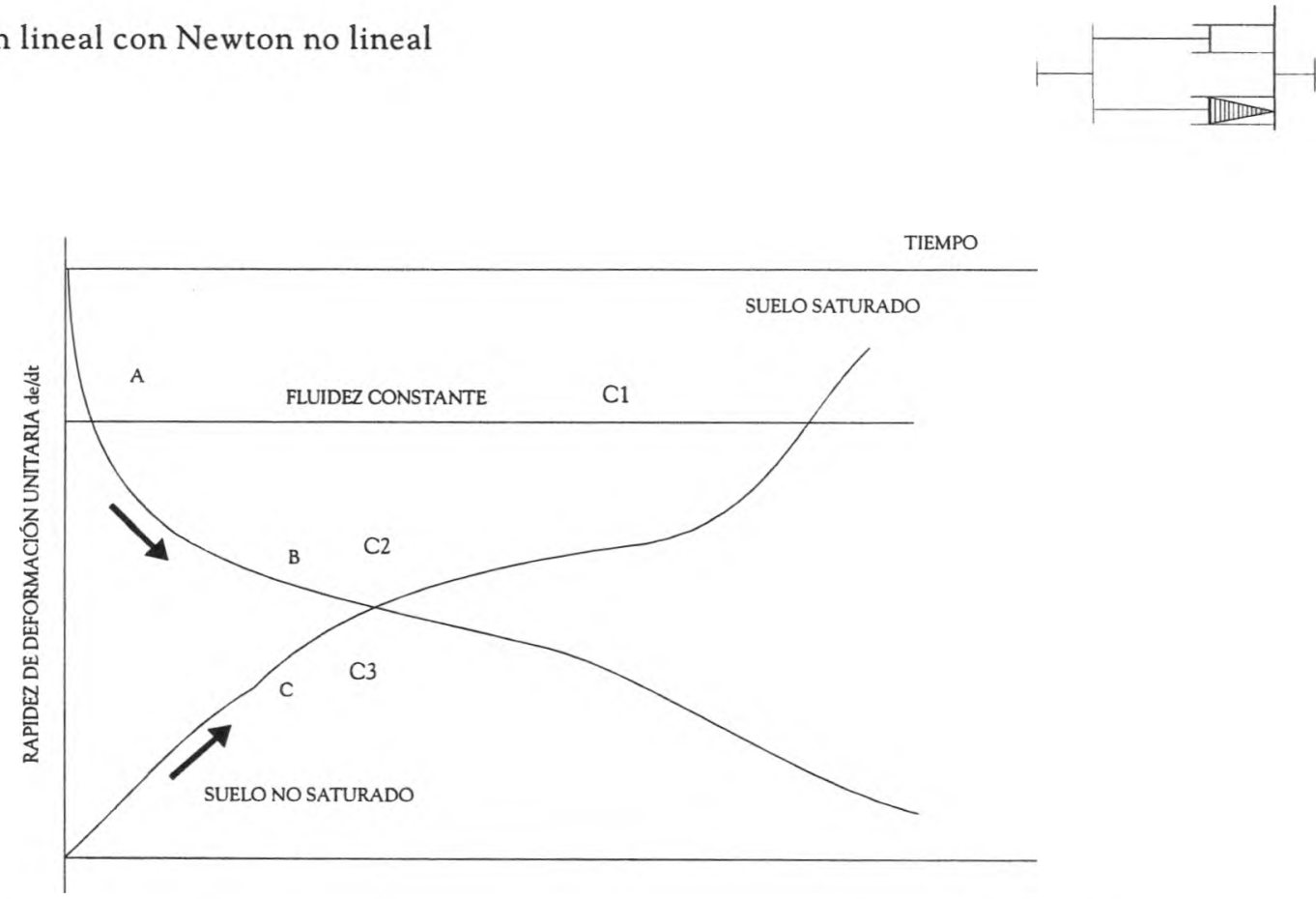

Figura 3. Configuración típica de las leyes Newtonianas con los parámetros de fluidez descritos 
Los parámetros del suelo $\mathrm{m}_{\mathrm{ep}}, \alpha$, b se determinan en el laboratorio en probetas de suelo inalterado.

La unidad reológica de Terzaghi (1941), tiene como base la unidad Lord Kelvin. La tabla 1 representa el proceso de consolidación de un suelo elasto-plástico saturado, pero no contempla el fenómeno de viscosidad intergranular. Que puede estudiarse en Zeevaert (1983).

Adicionando en serie a la unidad de Terzaghi con la unidad $Z$ en la figura 4 deducida por el autor, que representa el comportamiento retardado por la viscosidad intergranular de un suelo saturado, se obtiene la ecuación básica para el cálculo del proceso de consolidación del suelo, incluyendo el fenómeno de viscosidad intergranular (Zeevaert, 1985).

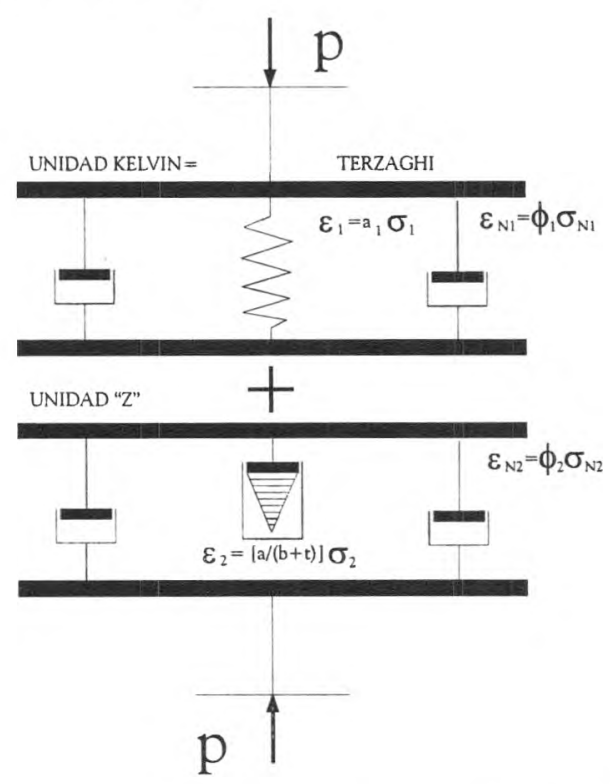

Figura 4. Unidades aplicables al fenómeno de consolidación

Para el suelo arcillo-limoso, doblemente floculento elasto-plástico y con viscosidad intergranular (Figura 4), el autor ha desarrollado la fórmula para poder pronosticar el hundimiento con el tiempo, por la carga sobre el suelo, sin rebasar su resistencia (Zeevaert, 1985).

$$
\mathrm{e}=\mathrm{m}_{\mathrm{ep}}(\Delta \mathrm{p})\left[\mathrm{F}\left(\mathrm{T}_{\mathrm{v}}\right)+(\beta) \log \left(1+\zeta \mathrm{T}_{\mathrm{v}}\right)\right]
$$

Los parámetros del suelo que intervienen $\mathrm{m}_{\mathrm{ep}}, \beta, \zeta$ y $\mathrm{c}_{\mathrm{v}}$ se determinan en el laboratorio con el odómetro en probetas de suelo inalterado.

Aquí:

$\varepsilon \quad$ Deformación unitaria

$\mathrm{m}_{\text {ep }}$ Módulo de compresibilidad volumétrico unitario elasto-plástico
$\mathrm{F}\left(\mathrm{T}_{\mathrm{v}}\right)$ Función del tiempo de la consolidación primaria, unidad reológiga de Terzaghi

$\mathrm{T}_{v}$ Factor tiempo en la consolidación de Terzaghi

$c_{v} \quad$ Coeficiente de consolidación, Terzaghi

$\mathrm{m}_{\mathrm{t}} \quad$ Módulo de compresibilidad volumétrica unitario de la viscosidad intergranular

$\beta \quad$ Relación de $\mathrm{m}_{\mathrm{t}} / \mathrm{m}_{\mathrm{ep}}$

$\zeta \quad$ Factor que modifica al factor tiempo de la unidad reológica Terzaghi-Zeevaert

Las fórmulas (1) y (2) han sido utilizadas por años y en múltiples ocasiones en la práctica profesional, con resultados satisfactorios.

Se utilizan para pronosticar la deformación del subsuelo en diferentes circunstancias físicas, principalmente por el hundimiento que provoca la carga sobre el suelo de los edificios. Zeevaert (1998) y (1985).

De lo expuesto anteriormente, se puede comprender la utilidad de la reología, ciencia física que ofrece el poder de establecer unidades reológicas con las leyes de la naturaleza para los suelos que representan el comportamiento básico natural.

Otras de las leyes de la física que se aplican al conocimiento del comportamiento de los suelos, es la de los gases que contiene el agua de poro del suelo en solución, o bien, en burbujas microscópicas; estas leyes son las de Gay-Lussac-Mariotte y la de Henry.

Por medio de estas leyes, se puede conocer la compresibilidad de la mezcla gas-agua que contenga el suelo, por lo que es importante tomarla en cuenta en los suelos saturados. Zeevaert (1982), (1988) y (1986).

La ley de Coulomb, acerca de la fuerza de atracción de las masas de dimensiones moleculares y de polos diferentes, suelo-agua, facilita la compresión del agua altamente viscosa que rodea a los granos de minerales de arcillas. El agua se adhiere a los granos con una intensidad que depende del inverso del cuadrado de la distancia entre las moléculas del agua y del grano, así como de la actividad del mineral de arcilla cerca de la periferia del grano, el agua llega a cristalizarse en capas moleculares y se conoce como agua sólida. Zeevaert (1983), (1997), (1998), (1985), (1941) y (1982).

Para poder admitir la combinación de las leyes que se utilizan para la obtención de una fórmula básica, es necesario verificar dichas fórmulas por medio de la observación con los fenómenos reales de campo y los parámetros que las rigen, los cuales son obtenidos en el laboratorio.

Es indispensable la investigación en el laboratorio de los parámetros, las pruebas para determinarlos deben efectuarse con probetas de suelo inalterado, las cuales deben ser representativas de las condiciones físicas estratigráficas de campo. 
En la mayoría de los casos se obtienen buenos resultados; sin embargo, el resultado depende muchas veces del valor que tengan las muestras utilizadas y la precisión de los ensayos en el laboratorio (Zeevaert, 2000).

\section{Conclusiones}

Finalmente, para llegar a una expresión o fórmula que represente cierto tipo de suelo, es indispensable que tenga como base el uso de las leyes naturales de la reología.

Durante la larga vida profesional y académica del autor, ha podido constatar que las fórmulas empíricas no tienen una base física científica, por consiguiente, no son confiables en su aplicación, excepto para utilizarse en la localidad en donde se registraron, pero no pueden proporcionar resultados cercanos a la realidad en forma incondicional, ya que los suelos en la naturaleza, aún con la misma constitución física, difiere los unos de los otros en su formación ambiental y así también los parámetros que los rigen.

Existen casos que con curvas de observación de un comportamiento específico en cierto lugar o en el laboratorio en probetas de un suelo, se obtienen fórmulas matemáticas ajustadas a dichas curvas, para que sirvan de forma generalizada en la práctica profesional; lo cual no es físicamente aceptable.

Es muy importante que el estudioso sepa diferenciar la realidad en estos casos, apoyándose en la reología física del problema de que se trate, relacionándolo con el comportamiento real del suelo en el campo.

\section{Agradecimientos}

El autor agradece al profesor Ing. Germán López Rincón sus comentarios y aportaciones durante el desarrollo del presente trabajo.

\section{Referencias}

Terzaghi K. (1941). Undisturbed Clay Samples and Undisturbed Clays. Journal of the Boston Society of Civil Engineers, No. 324.

Van-Nostrand R. (1983). Foundation Engineering for Difficult Subsoil Conditions. Segunda Edición.

Zeevaert-Wiechers L. (1982). Descompresión en depósitos de suelos impermeables. DEPFI-UNAM, México.

Zeevaert-Wiechers L. (1985). Consolidation in the Intergranular Viscosity of High Compressible Soils. ASTM Committe D-18 on Soil and Rock, Lauderdel, Florida, USA, enero.

Zeevaert-Wiechers L. (1986). Liquefaction Induced in the Sea Bed Sediments Due to Wave Action. 20 International Conference on Coastal Engineering, Taiwan, Republic of China, nòviembre. Reproducida por la División de Estudios Superiores de la Facultad de Ingeniería, UNAM, México, diciembre.

Zeevaert-Wiechers L. (1988). Seismo-Geodynamics of the Ground Surface and Building Foundations in Mexico City. DEPFI-UNAM. México.

Zeevaert-Wiechers L. (1994). Esfuerzo deformación tiempo en suelos finos no saturados. Revista Ingeniería, No. 9.

Zeevaert-Wiechers L. (1997). La investigación del suelo por su estructura y comportamiento reológico. Instituto Politécnico Nacional, México.

Zeevaert-Wiechers L. (1998). Compendio elemental de mecánica de suelos. DEPFI-UNAM, México.

Zeevaert-Wiechers L. (2000). Importancia de los parámetros en la aplicación de los problemas de mecánica y dinámica de los suelos. DEPFI-UNAM. México.

Zeevaert-Wiechers L. (2000). Comportamiento físico cuantitativo de los suelos finos colapsibles y residuales expansivos. Revista Ingeniería. Investigación y Tecnología. FI-UNAM, Vol. I, No.3,131-139.

\section{Bibliografía sugerida}

Zeevaert-Wiechers L. (1952). Compresibilidad de la arcilla volcánica de la ciudad de México. Congreso Científico del IV Centenario de la Universidad Nacional Autónoma de México, Vol. XXV, No.2.

Zeevaert-Wiechers L. (1952). Estratigrafía y problemas de ingeniería de los depósitos de arcilla lacustre de la ciudad de México. Congreso Científico del IV Centenario de la UNAM, Ingeniería y Arquitectura, Vol. XXX, Nos. 7,8,9.

Zeevaert-Wiechers L. (1953). Outline on the Mechanical Characteristics of the Unconsolidated Sedimentary Deposits in the Basin of the Valley of Mexico. IV Congreso "Inqua", Italia, Roma, septiembre.

Zeevaert-Wiechers L. (1957). Foundation Design and Behavior of Tower Latino Americana in Mexico City. Londres-Inglaterra. Geotechnique, The Institution of Civil Engineers, Vol.III, 188-189.

Zeevaert-Wiechers L. (1961). Grose Ausgleichsgründungen Auf Volkanischem Ton Mit Höher Zuzammendrückbarkeit in Mexico City. Baugrundkursus, Essen Germany.

Zeevaert-Wiechers L. (1987). Design of Compensated Foundations. Ground Engineers Reference Book, Edited by Bell F.G. Butterworths Scientific LTD, England.

Zeevaert-Wiechers L. (1993). Endurecimiento de las arcillas. Sociedad Mexicana de Mecánica de Suelos, México.

Zeevaert-Wiechers L. (1996). Módulos de deformación unitaria en arenas y limos en la interacción 
suelo-estructura de cimentaciones. Universidad Veracruzana, Facultad de Ingeniería, UNAM, México.
Zeevaert-Wiechers L. (1998). Análisis de la cimentación tipo Lez. DEPFI-UNAM, México.

\section{Semblanza del autor}

Leonardo Zeevaert-Wiechers. Obtuvo el título como ingeniero civil en 1939 en la Escuela Nacional de Ingenieros de la UNAM. Estudió el posgrado en el Instituto Tecnológico de Massachusetts donde recibió el grado de maestro en ingeniería en 1940. En 1943 inició una estrecha colaboración con el Dr. Karl Terzaghi en una investigación acerca de la estabilidad de las cortinas de corazón hidráulico construidas en México a principios del siglo XX; esto lo llevó en 1947 a trabajar en problemas especiales de mecánica de suelos en la Universidad de Illinois, donde obtuvo el grado de doctor (Ph. D) en 1949. Ha recibido numerosos reconocimientos, entre ellos: la medalla de oro profesional otorgada por el Instituto Americano de Arquitectos, diploma a la innovación tecnológica, designación de profesor emérito en la UNAM y miembro de la Academia Nacional de Ingeniería de EUA, entre otros. El buen comportamiento, la cimentación y estructura de obras de ingeniería que ha diseñado, entre ellas la Torre Latinoamericana, en donde introdujo el concepto de flexibilidad controlada en edificios altos, le han valido para su reconocimiento a nivel internacional. Ha escrito 160 artículos, una gran cantidad de libros y ha presentado ponencias relacionadas con mecánica de suelos, ingeniería de cimentaciones y sísmica de cimentaciones. 
